\title{
A mild variant of cystic fibrosis
}

\author{
Eric W F W Alton \\ Ion Transport Unit, National Heart and Lung Institute, London, UK
}

Introductory article

\section{A cystic fibrosis mutation associated with mild lung disease}

\author{
K-H Gan, HJ Veeze, MW van den Ouweland, DJJ Halley, H Scheffer, A van der Hout, SE Overbeek, \\ J C de Jongste, W Bakker, HGM Heijerman
}

Background. Cystic fibrosis is the most common lethal autosomal recessive disorder among whites. Among Dutch patients with cystic fibrosis, $4 F 508$ is the most common mutation and A455E the second most common mutation of the cystic fibrosis transmembrane conductance regulator gene on chromosome 7. A455E is associated with preserved pancreatic function and residual secretion of chloride across membranes. We investigated whether it is also associated with less severe pulmonary disease in patients with cystic fibrosis. Methods. A total of 33 patients with compound heterozygosity for the A455E mutation were matched according to age and sex with patients who were homozygous for the 4F508 mutation. The pairs were analyzed with respect to the following outcome variables: age at diagnosis, pulmonary-function values, and the frequency of pseudomonas colonization, pancreatic sufficiency, and diabetes mellitus. Results. Cystic fibrosis was diagnosed at a later age in the patients with the A455E mutation than in the $\triangle F 508$ homozygotes (mean age at diagnosis, 15.0 vs. 3.1 years; $P<0.001)$. Fewer patients with the A455E mutation had pancreatic insufficiency (21.2 percent vs. 93.9 percent, $P<0.001)$, and none had diabetes mellitus ( 0 percent vs. 27.3 percent, $P=0.004$ ). Forced expiratory volume in one second (FEV 1 ) and forced vital capacity (FVC) were significantly higher in the patients with the A455E mutation (mean FEV $1,73.9$ percent of the predicted value vs. 54.3 percent of the predicted value; $P=0.002$; mean FVC, 88.7 percent of the predicted value vs. 76.3 percent of the predicted value; $P=0.04$ ). Fewer patients with the A455E mutation were colonized with Pseudomonas aeruginosa (33.3 percent vs. 60.6 percent, $P=0.02$ ). Conclusions. A455E is a common mutation causing cystic fibrosis in The Netherlands. Although several mutations are known to be associated with less severe pancreatic disease, our findings demonstrate a correlation between the A455E mutation and mild pulmonary disease. Because mortality in this disease depends primarily on the progression of pulmonary disease, patients with the A455E mutation have a better prognosis than patients who are homozygous for the $\Delta F 508$ mutation. (N Engl J Med 1995;333:95-9)

The article by Gan et al provides the first important evidence that there is a genotypic correlation with phenotype in the lungs of patients with cystic fibrosis. ${ }^{1}$ This finding will be considered in the light of (1) the basic abnormality in cystic fibrosis, (2) how the more than 400 mutations now identified in the cystic fibrosis gene relate to this basic abnormality, (3) the data prior to this publication regarding the link of these mutations with clinical disease, and (4) how the information provided in this paper alters this information.

Cystic fibrosis: the basic defect

The cystic fibrosis (CF) gene on chromosome 7 codes for a protein entitled cystic fibrosis transmembrane conductance regulator (CFTR). ${ }^{2}$ Numerous studies have shown that this protein acts as a chloride channel in the epithelium of the respiratory and intestinal tracts as well as in other epithelial-lined organs. ${ }^{3}$ Whilst the precise function of chloride transport in these organs is unclear, the most probable current hypothesis relates to the concomitant movement of water that will osmotically follow chloride secretion. Thus, for example, in the airway epithelium, chloride movement from the inside of the cell on to the airway surface will be followed by water (fig 1A), and it is probable that this water contributes importantly to the sol layer component of the airway surface liquid. In subjects with cystic fibrosis the CFTR chloride channel does not function normally (fig 1B), leading to reduced water movement, an altered sol layer and, consequently, impaired mucociliary clearance. This represents one way in which impaired chloride secretion may relate to the recurrent infection to which these patients are susceptible.

However, it is important to mention two other possible links to pathogenesis. Firstly, both in the upper and lower airways of these patients the absorption of sodium ions from the mucosal surface into the cell is increased compared with subjects without cystic fibrosis (fig 1B). ${ }^{4}$ 


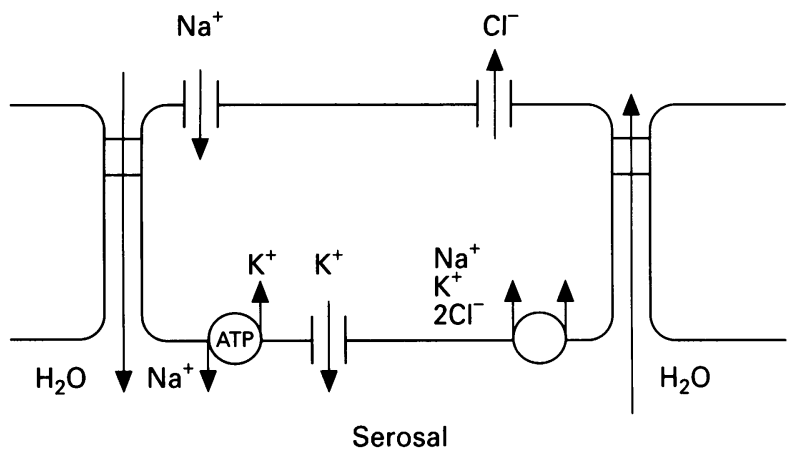

B

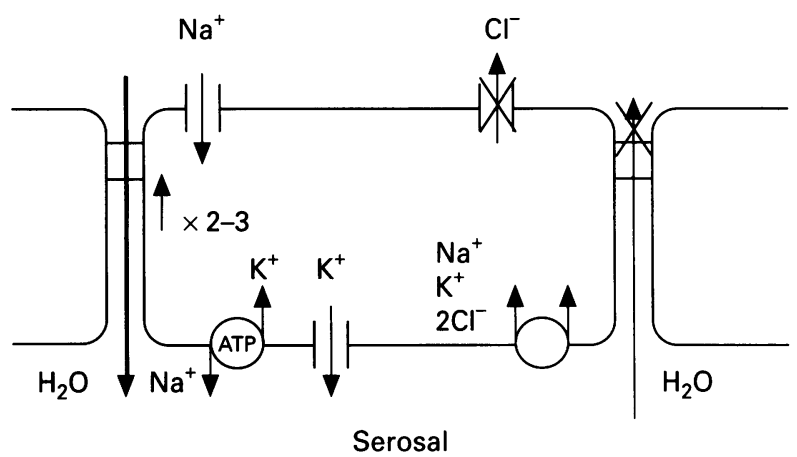

Figure 1 Diagram of airway epithelial cell showing $(A)$ the ion transport processes in a normal subject and $(B)$ the ion transport abnormalities present in cystic fibrosis.

This leads, by osmosis, to increased absorption of water and will further exacerbate the problem caused by the reduced chloride secretion on to the mucosal surface. The link between this secondary abnormality and the primary defect is uncertain but there is compelling evidence that both abnormalities are present. ${ }^{5}$ Secondly, it is worth noting that the link between altered chloride transport and increased respiratory infection is not necessarily limited to altered mucociliary clearance. Three separate links have recently been made between cystic fibrosis and altered clearance of Pseudomonas aeruginosa. Firstly, the binding site for $P$ aeruginosa has been shown to be an asialoGM-1 receptor in a number of cell types. ${ }^{6}$ These asialoGM-1 receptors have been shown to be upregulated in cystic fibrosis, although how this relates to the chloride abnormality is at present uncertain. Thus, both cultured and freshly obtained respiratory epithelial cells from patients with cystic fibrosis show increased binding of $P$ aeruginosa compared with those from patients without cystic fibrosis, and we have recently shown that transfer of a normal copy of the CFTR gene into these cells can reduce this binding towards normal levels. Secondly, a study has indicated that one function of the respiratory epithelium is to ingest bacteria followed by sloughing of the cell. ${ }^{7}$ This process was shown to be defective in patients with cystic fibrosis, suggesting a mechanism by which increased Pseudomonas colonisation may occur. Finally, a recent paper has shown that the airway epithelium is likely to produce defensin-like molecules which facilitate bacterial killing. ${ }^{8}$ Because of the ion transport abnormality it is likely that the airway surface fluid has an altered composition in subjects with cystic fibrosis, with increased levels of both sodium and chloride. This paper demonstrated that, although normal levels of the de-

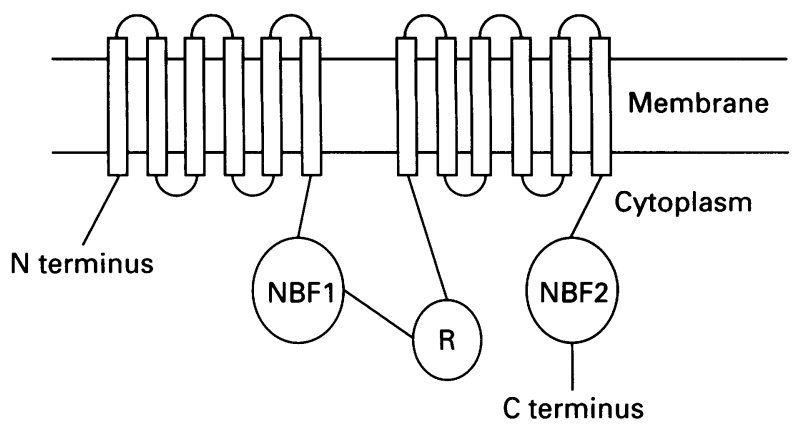

Figure 2 Diagrammatic representation of the predicted structure of cystic fibrosis transmembrane conductance regulator (CFTR). It consists of two hydrophobic membrane spanning domains each composed of six transmembrane segments, two nucleotide binding folds (NBF1 and NBF2), and a large cytoplasmic domain known as the $R$ unit.

fensin-like molecules are produced in cystic fibrosis, they do not appear to function normally with respect to killing $P$ aeruginosa in the presence of these increased concentrations of salt. This abnormality could be reversed by returning the sodium and chloride concentrations towards normal levels. Thus, mutations in the CFTR gene cause a variety of malfunctions and the sequence of events from altered chloride transport to respiratory failure is only gradually being pieced together.

\section{Link between CFTR mutations and altered chloride transport}

To date, over 400 mutations have been identified in the CFTR gene which have been conveniently classified into four categories with respect to the chloride defect. ${ }^{9}$ Class 1 mutations include those in which it is unlikely that any CFTR protein will be produced. These relate to nonsense and frameshift mutations, or those producing premature termination of the protein. Examples include G542X, a mutation with a frequency of around $3 \%$ worldwide. Class 2 mutations are characterised by the production of a mutant protein which is recognised by the quality control mechanism in the cell, and consequently not allowed to traffic to the normal site at the mucosal surface of the cell. Delta F508, the commonest mutation found in approximately $70 \%$ of patients with cystic fibrosis, is an example of this class. Whilst the majority of the abnormal protein is filtered out by the chaperone system (principally the HSP70 chaperone), ${ }^{10}$ a small percentage, estimated at $1-2 \%$, may reach the apical membrane and produce some residual function in these mutations. Class 3 mutations include those in which the protein reaches the apical membrane but does not undergo normal regulation through intracellular ATP. Thus, it is known that for CFTR to open normally it requires binding of intracellular ATP to two intracellular portions of the molecule known as nucleotide binding folds 1 and 2 (fig 2). Type 3 mutations prevent the normal opening of the channel upon binding of ATP. Thus, channel open probability - that is, the time that the channel spends open - is reduced. An example of this type of mutation is G551D with a worldwide frequency of approximately $3 \%$. Finally, class 4 mutations have an abnormality in the portion of the channel through which the chloride is conducted. Thus, this protein reaches the apical membrane, does not show alterations in open probability, but during the time the channel is open does not conduct chloride at the normal rate. Such mutations are relatively rare and are ex- 
emplified by $\mathrm{R} 117 \mathrm{H}$. It should be evident from this brief summary that class 3 and 4 mutations are likely to show an increased level of chloride channel function over class 1 and 2 mutations; this has been confirmed at the single channel level using the patch clamp technique. ${ }^{11}$

\section{Correlation of different CFTR mutations with clinical disease}

In parallel with the increased information regarding the different classes of CFTR mutations obtained in the laboratory, data have been collected with regard to genotype/phenotype correlations. A large number of studies have been published in an attempt to assess this relationship, and a number of broad principles can now be discerned. Firstly, it is clear that it is important to separate intestinal from respiratory disease when considering such genotype/phenotype correlations. Soon after cloning of the CF gene in 1989 it became evident that the commonest mutation, $\Delta \mathrm{F} 508$, is associated with pancreatic insufficiency ${ }^{12}$ - that is, most of these patients require pancreatic supplements. As data have been gradually collected it is evident that type 1 and 2 mutations, in which relatively little protein is produced, are associated with pancreatic insufficiency. In contrast "milder" mutations such as $\mathrm{R} 117 \mathrm{H}$ (class 4) are generally associated with pancreatic sufficiency. ${ }^{13}$ These findings follow quite well from the previous description of the function of the CFTR molecule. Interestingly, this correlation with intestinal function is also carried through into the recently generated mouse models of cystic fibrosis. Thus, "null" mice which produce no CFTR protein, equivalent to a type 1 mutation, show marked intestinal problems resulting from a condition similar to meconium ileus, which result in the death of these animals early in their life time. In contrast, the recently generated G551D mouse (type 3 mutation) shows a markedly reduced incidence of such intestinal blockage and consequently prolonged survival.

Until the publication of the paper by Gan et al the available data showed that, with respect to lung disease, there was, however, no correlation between genotype and phenotype. ${ }^{14}$ This was irrespective of whether the mutation was, for example, a class 4 "mild" mutation, the "mild" relating to pancreatic disease but not to lung disease. These findings of a lack of correlation between genotype and phenotype in the lung have been confirmed in a large number of studies. The reasons for this discrepancy between the respiratory and intestinal tracts are not clear, but include a number of possibilities. One important feature is that, in the intestinal tract, CFTR represents the predominant and, in some cases, the only chloride channel present. To correlate with this, the level of CFTR protein is relatively higher in the intestine than in the respiratory tract. ${ }^{15}$ In contrast, in the airways it is clear that there are a number of different chloride channels present, some of which are not regulated through an intracellular increase in cAMP, the mediator that regulates CFTR. Secondly, as noted above, sodium absorption may also play a part in disease pathogenesis in the lungs. Whilst some studies have shown increased sodium absorption in the small intestine of patients with cystic fibrosis, ${ }^{16}$ this is not a consistent finding. Further, as noted above, colonisation with Pseudomonas clearly plays an important part in disease progression. This and other environmental factors may be crucial to the differences between the intestinal and respiratory tracts. Finally, the expression of genes other than that for CFTR may also have a role
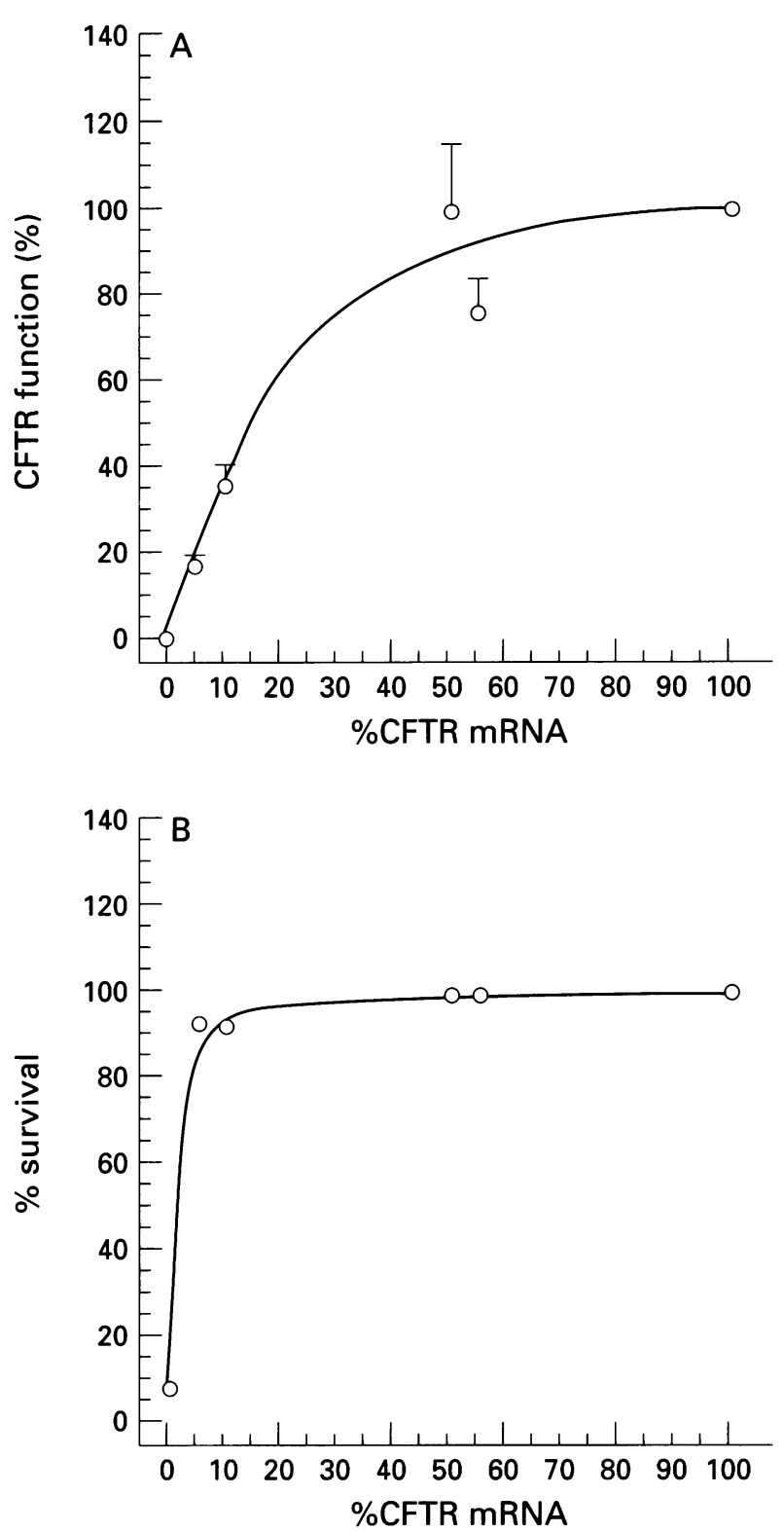

Figure 3 Relationship between levels of normal CFTR MRNA and (A) chloride secretion or (B) survival. Values are normalised to the range provided by normal mice $(100 \%)$ and $C F$ null mice $(0 \%)$.

in disease pathogenesis, and such expression may be organ-specific. In summary, therefore, the role of CFTR in the airways may be less directly related to chloride transport than in the intestinal tract, and involve linkage with a number of different steps along the pathway of disease pathogenesis. This may, in turn, relate to the absence of genotype/phenotype correlations within the lung.

\section{Novel findings of the paper by Gan et al}

The key finding of the paper by Gan et $a l^{1}$ is the identification of a mutation, A455E, that does appear to correlate with better lung prognosis. The mutation is clearly a "mild mutation" with respect to intestinal disease and, indeed, the patients in this study showed a markedly reduced degree of pancreatic insufficiency compared with $\Delta \mathrm{F} 508$ homozygotes. Lung function was significantly higher in age and sex matched patients than in $\Delta \mathrm{F} 508$ homozygotes, fewer patients were colonised with Pseudomonas, and disease was diagnosed at a later age in the subjects with $\mathrm{A} 455 \mathrm{E}$ mutation. As 


\section{LEARNING POINTS}

* The CF gene product CFTR functions as a chloride channel in epithelial cells.

* The link between CFTR dysfunction and disease pathogenesis is unclear; altered mucociliary clearance and/or changes in bacterial clearance may be key mechanisms. * Several hundred CF mutations have been identified and produce varying levels of CFTR
chloride function.

\section{* Correlations between this function and disease severity have previously been demonstrated in the intestinal tract.}

* The paper by Gan et al provides the first evidence for such a correlation for the lung.

noted above, such findings have not so far been identified and are obviously important for a number of reasons. Firstly, from the viewpoint of the patients and their relatives, identification of this mutation may suggest an improved prognosis. Clearly, however, the validity of such a prediction is difficult to gauge until larger numbers have been assessed, and it may be prudent to await such studies before placing overemphasis on information given to patients. Further, whilst the mutation is relatively common in Holland (around $7 \%$ ), being second only in prevalence to $\Delta \mathrm{F} 508$, the frequency worldwide is probably less than $1 \%$. Secondly, from a mechanistic point of view it will be important to understand why the $\mathrm{A} 455 \mathrm{E}$ mutation differs from others in predicting a favourable outcome. The ameliorating effects of this mutation may occur at one or more of the interactions of CFTR noted above. Thus, it will be important to know if the level of sodium absorption in the lungs of these subjects differs from that seen with other mutations, and to establish whether the number of asialoGM1 receptors or the composition of the airway surface liquid differs. One final consideration relates to the level of chloride secretion present in patients with the A455E mutation, which has been shown to allow residual secretion of chloride. ${ }^{17}$ The level of chloride secretion required to prevent disease is clearly a key question, both with respect to genotype/phenotype relationships and the degree of correction required in gene therapy studies. To begin to address this issue we have interbred different types of CF mice and wild-type animals. ${ }^{18}$ In this way, mice with differing levels of wild-type CFTR mRNA were produced, and these levels were assessed for their correlation with both chloride secretion and pathology/survival. It is important to note that, in these mice, the latter relates to intestinal rather than lung disease. Figure 3 shows that the relationship is not linear, with small increases in CFTR mRNA producing disproportionately larger increases in both chloride secretion and, in turn, survival. A455E may produce a small increase in function above a critical level, resulting, in turn, in a disproportionately large increase in function sufficient to influence lung disease. Finally, it is worth noting that, given the relative rarity of some mutations, A455E may not turn out to be the only mutation associated with an improved respiratory prognosis.

1 Gan K-H, Veeze HJ, van den Ouweland AMW, Halley DJJ, Scheffer $\mathrm{H}$, van der Hout A, et al. A cystic fibrosis mutation associated with Hild van der Hout A, et al. A cystic fibrosis muta

2 Riordan JR, Rommens JM, Kerem BS, Alon N, Rozmahel R, Grzelczak $\mathrm{Z}$, et al. Identification of the cystic fibrosis gene: cloning and characterization of complementary DNA. Science 1989;245:1066-73.

3 Welsh MJ. Electrolyte transport by airway epithelia. Physiol Rev 1987; 67:1143-84.

4 Knowles M, Gatzy J, Boucher R. Increased biolectric potential difference across respiratory epithelia in cystic fibrosis. $N$ Engl f Med 1981;305: $1489-95$.

5 Stutts MJ, Canessa CM, Olsen JC, Hamrick M, Cohn JA, Rossier BC, et al. CFTR as a cAMP-dependent regulator of sodium channels. Science $1995 ; 269: 847-50$.

6 Imundo L, Barasch J, Prince A, Al-Awqati Q. Cystic fibrosis epithelial cells have a receptor for pathogenic bacteria on their apical surface. Proc Natl Acad Sci USA 1995;92:3019-23.

7 Pier GB, Grout M, Zaidi TS, Olsen JC, Johnson LG, Yankaskas JR, et al. Role of mutant CFTR in hypersusceptibility of cystic fibrosis patients to lung infections. Science 1996;271:64-7.

8 Smith JJ, Travis SM, Greenberg EP, Welsh MJ. Cystic fibrosis airway epithelia fail to kill bacteria because of abnormal airway surface fluid. Cell 1996;85:229-36.

9 Welsh MJ, Smith AE. Molecular mechanisms of CFTR chloride channel dysfunction in cystic fibrosis. Cell 1993;73:1251-4.

10 Yang Y, Janich S, Cohn JA, Wilson JM. The common variant of cystic fibrosis transmembrane conductance regulator is recognised by hsp 70 and degraded in a pre-Golgi nonlysosomal compartment. Proc Natl 70 and degraded in a pre-Golgi non

11 Sheppard DN, Rich DP, Ostedgaard LS, Gregory RJ, Smith AE, Welsh MJ. Mutations in CFTR associated with mild-disease-form C1-channels with altered pore properties. Nature 1993;362:160-4.

12 Kerem E, Corey M, Kerem BS, Rommens J, Markiewicz D, Levison $\mathrm{H}$, et al. The relation between genotype and phenotype in cystic fibrosis - analysis of the most common mutation. N Engl $\mathscr{f}$ Med 1990; 323:1517-22.

13 Fitzgerald D, Van Asperen P, Henry R, Waters D, Freelander M, Wilson $M$, et al. Delayed diagnosis of cystic fibrosis in children with a rare genotype (delta F508/R117H). F Paediatr Child Health 1995;31: 168-71.

14 Anonymous. Correlation between genotype and phenotype in patients with cystic fibrosis. $N$ Engl F Med 1993;329:1308-13.

15 Crawford I, Maloney PC, Zeitlin PL, Guggino WB, Hyde SC, Turley $\mathrm{H}$, et al. Immunocytochemical localization of the cystic fibrosis gene product CFTR. Proc Natl Acad Sci USA 1991;88:9262-6.

16 Baxter P, Goldhill J, Hardcastle J, Hardcastle PT, Taylor CJ. Enhanced intestinal glucose and alanine transport in cystic fibrosis. Gut 1990; intestinal gluco

17 Veeze HJ, Halley DJJ, Bijman J, de Jongste JC, de Jonge HR, Sinaasappel $M$. Determinants of mild clinical symptoms in cystic fibrosis patients: residual chloride secretion measured in rectal biopsies in relation to the genotype. $\mathcal{F}$ Clin Invest 1994;93:461-6.

18 Dorin JR, Farley R, Webb S, Smith SN, Farini E, Delaney SJ, et al. A demonstration using mouse models that successful gene therapy for cystic fibrosis requires only partial gene correction. Gene Therapy 1996 (in press). 